CLINICAL REVIEW

\title{
Radiological changes in hepatic amoebiasis
}

\author{
S. RAMACHANDRAN \\ M.D., M.R.C.P., M.R.C.P.E. \\ Physician
}

\author{
D. L. N. JAYAWARDENA \\ M.B.B.S., D.M.R.D.(Lond.) \\ Visiting Radiologist
}

\author{
J. R. A. Perumal \\ M.B.B.S. \\ Medical Officer
}

The Government Hospital, Negombo, Ceylon

\section{Summary}

Radiological abnormalities were observed in $88.3 \%$ of patients with the syndrome of hepatic amoebiasis. While this figure is higher than those reported in previous studies, it appears that it is due to the large number of patients who showed an elevation of the diaphragm in the right lateral views of the chest. It was observed that a proportion of the patients with an elevation of the right dome of the diaphragm in the lateral views showed no significant elevation in the postero-anterior views, these patients constituting a radiologically distinct group. It is thus important that lateral views of the chest should be performed routinely in all cases of suspected hepatic amoebiasis.

Cases of proved hepatic abscesses also conform to one or other of the radiological groups described. Abscesses situated inferiorly or anteriorly in the liver could be associated with an elevation of the right dome of the diaphragm.

While chest radiology is of importance in the diagnosis of the syndrome of hepatic amoebiasis, it is not peculiar to this syndrome alone and is of only relative diagnostic value in clinically differentiating the cases where pus may be demonstrated from the cases where pus may not be demonstrable.

\section{Introduction}

The importance of radiology in the diagnosis of the syndrome of hepatic amoebiasis has been recognized since the descriptions of Munk (1944) and sub- sequently, radiological abnormalities were included as one of the criteria for the diagnosis of the syndrome (Lamont \& Pooler, 1958). The frequency of the abnormal findings in chest X-rays varied markedly in different series, the figures ranging from $64.8 \%$ to as much as $83.5 \%$. The commonest abnormality seen was an elevation of the right dome of the diaphragm, the frequency of which ranged from 27.5 to $74.3 \%$ in the larger studies reported (Table 1) The cause for the wide discrepancy in these figures, while obscure, may in part be due to the geographical distribution and varying patterns in the manifestations of the syndrome.

The purpose of this study was to evaluate the frequency and the type of abnormalities seen in chest radiology in cases of uncomplicated hepatic amoebiasis as it occurs in Ceylon and to detail any changes or patterns that may hitherto have not been sufficiently emphasized.

\section{Patients, materials, and methods}

One hundred and twenty cases of uncomplicated hepatic amoebiasis admitted to the medical unit of the Government Hospital, Negombo, Ceylon, were subjected to routine chest radiology. Both standard postero-anterior (PA) and right lateral teleradiograms of the chest were done in all the cases on admission to hospital.

The criteria for the diagnosis of the syndrome were a modification of those laid down by Kean (1955),

TABLE 1.

\begin{tabular}{lccc}
\hline \multicolumn{1}{c}{ Authors } & $\begin{array}{c}\text { Cases } \\
\text { studied }\end{array}$ & $\begin{array}{c}\text { Radiological } \\
\text { abnormalities } \\
(\%)\end{array}$ & $\begin{array}{c}\text { Elevation of } \\
\text { the diaphragm } \\
(\%)\end{array}$ \\
\hline Lamont \& Pooler (1958) & 242 & $83 \cdot 5$ & 69.4 \\
Islam et al. (1960) & 48 & $71 \cdot 8$ & $56 \cdot 1$ \\
Rajasuriya \& Nagaratnam (1962) & 91 & $64 \cdot 8$ & $\mathbf{2 7 . 5}$ \\
Amarjit Singh \& Jolly (1962) & 35 & 74.3 & $\mathbf{7 4 . 3}$ \\
Turrill \& Burnham (1966) & 100 & 66.6 & No details \\
Present series (1970) & 120 & 88.3 & 83.3 \\
\hline
\end{tabular}


they were: (1) an enlarged tender liver, (2) a previous history of diarrhoea with blood and mucus or an illness suggestive of hepatic amoebiasis, (3) suggestive haematological changes, (4) suggestive radiological changes and (5) a response to specific amoebicidal therapy. All the cases included in the study satisfied at least three of the above five criteria. No attempt was made during the study to separate the cases where pus was demonstrated from the cases where no pus was demonstrated.

\section{Results}

The individual radiological abnormalities seen in our cases are summarized in Table 2. They were:

\section{(1) Elevation of the right dome of the diaphragm in}

\section{$P A$ views}

Elevation of the right dome of the diaphragm in the PA view was seen in $55.8 \%$ of cases. This elevation was of a moderate or severe degree. By moderate is meant an elevation of between 1 and $2 \mathrm{in}$. above the level of the left dome of the diaphragm provided there is no elevation of the latter by gaseous distention of the stomach. 'Severe degrees of elevation' denotes an upward doming of the right diaphragm of over 2 in. above the left side. In a small proportion of cases $(5.8 \%)$ elevation of the diaphragm was associated with visible pleural reactions.

\section{(2) Elevation of the right dome of the diaphragm in} right lateral views

Elevation of the diaphragm either localized or involving the whole of the diaphragm was most striking in the lateral views, it being noted in $83.3 \%$ of the cases. Once again it varied in its degree, being either moderate or severe. When the diaphragmatic elevation was localized it always involved the anterior or the middle third of the dome. Elevations of the posterior part of the diaphragm were not encountered. It was an interesting observation that about $30 \%$ of these patients who showed an elevation in the right diaphragm either localized or generalized in the lateral views did not show this change in the postero-anterior views.

\section{(3) Pleural reactions}

Evidence of minor pleural reactions were seen in $7.5 \%$ of the cases, manifesting as small effusions or pleural thickening. In $5.8 \%$ of the cases it was associated with varying degrees of diaphragmatic elevation.

\section{(4) Pulmonary changes}

Changes in the lung parenchyma were noted in only $5 \%$ of the cases, they were consolidation with or without effusion or changes suggestive of bronchiectasis.

\section{(5) Absence of radiological changes}

No radiological abnormality was seen in $11.7 \%$ of the cases. Thus $88.3 \%$ of the cases in this study showed some radiological abnormality in the chest $\mathrm{X}$-rays as a consequence of hepatic amoebiasis.

Further observations revealed that these cases could be grouped into one of five fairly clear-cut radiologically distinct patterns depending on the combination of the various abnormalities noted (Table 3). They were:

Group 1. This included a proportion of cases, $50 \%$, who showed a significant elevation of the right dome of the diaphragm in both the PA and the right lateral views, with no evidence of either pleural or pulmonary changes. The elevation seen in the lateral views were often more striking than that seen in the PA views (Fig. 1a and $b$ ).

Group 2. This consisted of an interesting group o\$ $25.8 \%$ who showed absence of elevation of the righ dome of the diaphragm in the PA views but showe significant elevation of the anterior or the middle third of the diaphragm in the right lateral views (Fig. 2a and b).

Group 3. This group consisted of $7.5 \%$ of the cases who showed evidence of pleural reactions. $5 \cdot 8 \%$ showed a pleural reaction with elevations of the diaphragm in the PA and/or the lateral views (Fig. 3a and $b$ ) and the remaining $1.7 \%$ had only pleural reactions with no elevation of the diaphragm.

Group 4. This consisted of $5.0 \%$ of cases with prominent pulmonary changes with or without elevation of the diaphragm.

Group 5 . The $11.7 \%$ of cases where no radiological abnormalities were present.

Hepatic abscesses. Pus was demonstrated subsequent to $X$-ray examination in twelve cases $(10 \%)$. Eight of these patients showed a Group 1 type of radiological pattern while three had a Group 2

TABLE 2.

\begin{tabular}{lr}
\hline \multicolumn{1}{c}{ Radiological abnormality } & Cases (\%) \\
\hline Elevation of the right dome of the diaphragm-PA view & $55 \cdot 8$ \\
Elevation of the right dome of the diaphragm-right lateral views & $83 \cdot 3$ \\
Pleural reactions & $7 \cdot 5$ \\
Pulmonary changes & $5 \cdot 0$ \\
Abscence of radiological changes & $11 \cdot 7$ \\
\hline
\end{tabular}


TABLE 3.

\begin{tabular}{clr}
\hline $\begin{array}{c}\text { Radiological } \\
\text { group }\end{array}$ & \multicolumn{1}{c}{$\begin{array}{c}\text { Radiological } \\
\text { abnormality }\end{array}$} & $\begin{array}{c}\text { Cases } \\
(\%)\end{array}$ \\
\hline 1 & $\begin{array}{l}\text { Elevation of the right dome of the diaphragm in both } \\
\text { PA and lateral views }\end{array}$ & $50 \cdot 0$ \\
2 & $\begin{array}{l}\text { Elevation of the right dome of the diaphragm in the } \\
\text { lateral views, with no elevation in the PA views }\end{array}$ & 25.8 \\
& Pleural reactions with diaphragmatic elevation & 5.8 \\
$3($ a) & Pleural reactions with no diaphragmatic elevation & 1.7 \\
$3($ b) & Dominant pulmonary changes & $5 \cdot 0$ \\
5 & No changes & 11.7 \\
\hline
\end{tabular}
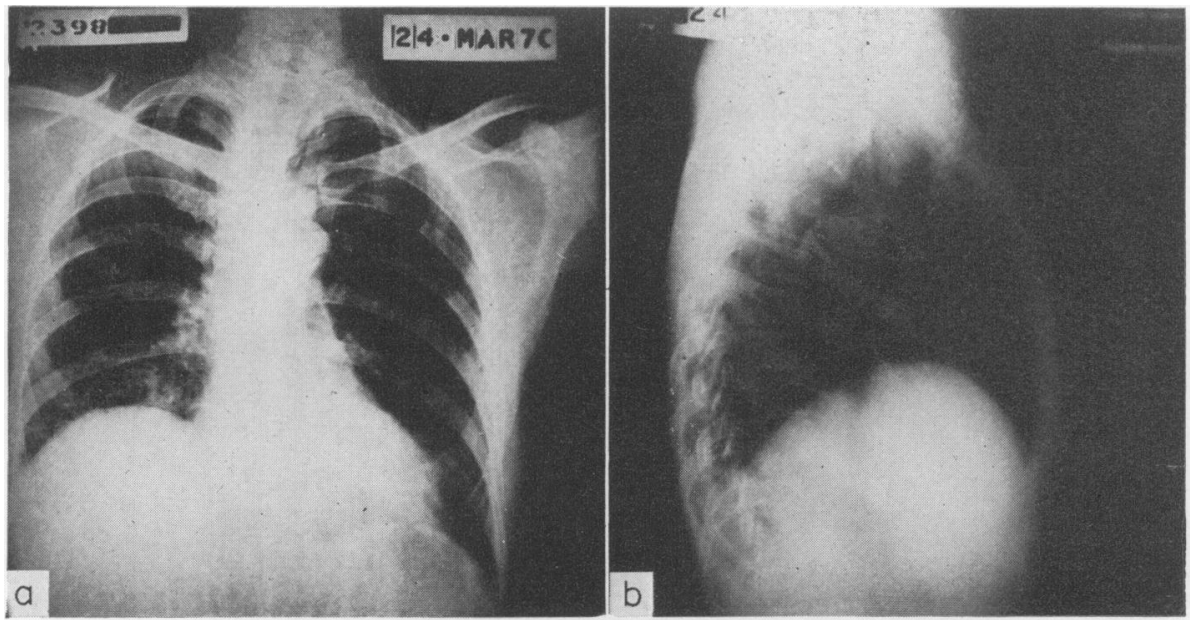

Fig. 1. Group 1 pattern, showing marked elevation of the diaphragm. (a) PA view, (b) right lateral view.
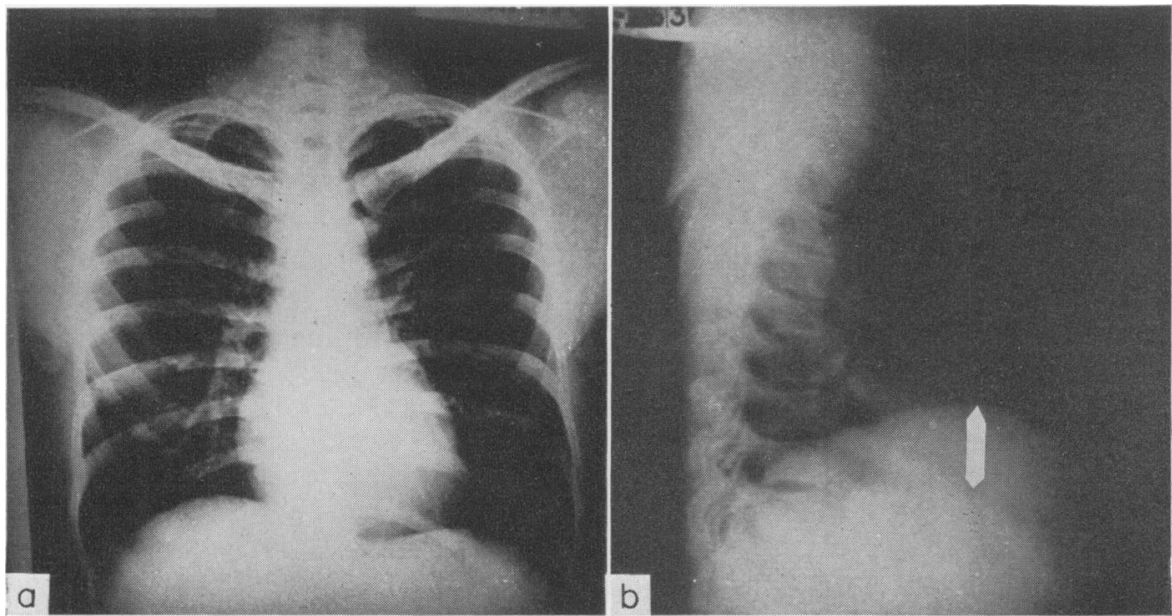

FIG. 2. Group 2 pattern, showing (a) no significant elevation of the diaphragm in the PA view, (b) significant elevation of the diaphragm in the right lateral view. 


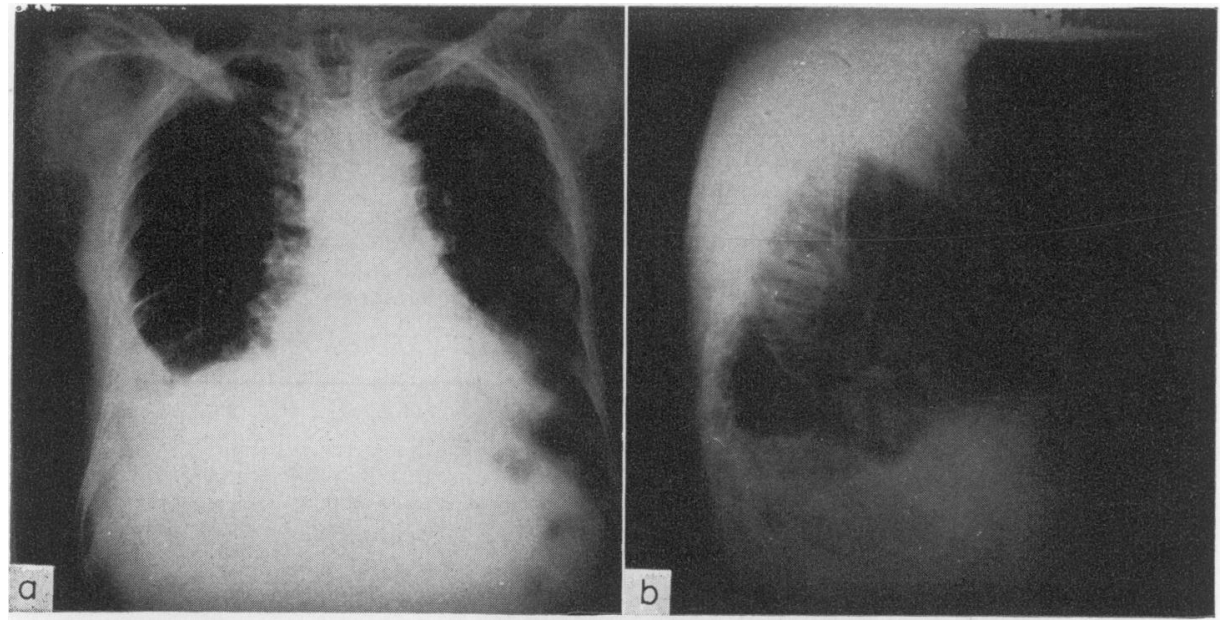

Fig. 3. Group 3 pattern, showing pleural reaction with an elevation of the diaphragm. (a) PA view, (b) right lateral view.
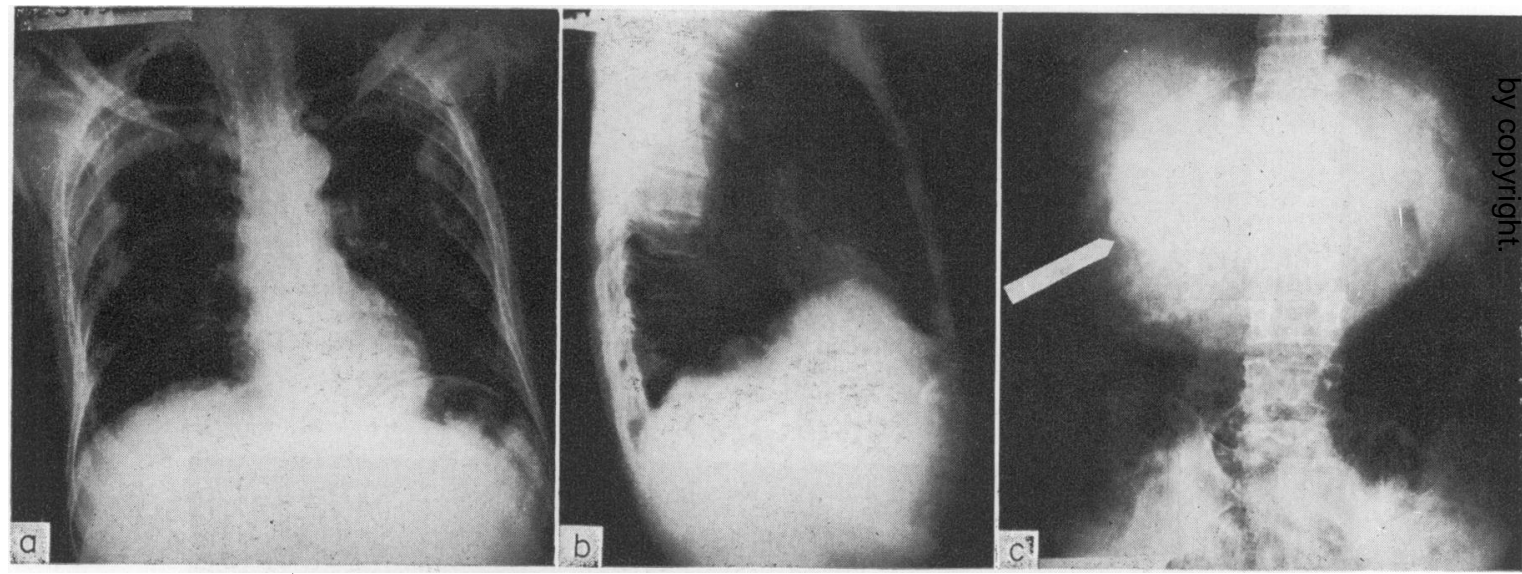

FIG. 4. Patient M.A., showing (a) no significant elevation of the diaphragm in the PA view, (b) significant elevation in the diaphragm in the right lateral view (Group 2). (c) Dye injected into the abscess cavity after aspiration, showing the position of the abscess.

pattern, the remaining patient having a Group 4 pattern. It thus appears that the cases of proven abscesses also conformed to the same radiological types as the cases where no pus was demonstrated.

The following case reports draw attention to some of the radiological features of hepatic abscesses which will be discussed.

(a) Patient M.A. had a large epigastric lump which on aspiration yielded two pints of chocolate pus. He showed a Group 2 radiological pattern, with no elevation of the diaphragm in the PA view and significant elevation of the diaphragm anteriorly in the right lateral view (Fig. 4a, b, and c).

(b) Patient M.A.I. had an abscess pointing into the right loin. His radiological pattern was of a Group 1 type with moderate elevation of the diaphragm in the PA view and more prominent elevation in the lateral view (Fig. 5a). Dye injected into the abscess cavity after aspiration showed that it was placed posteroinferiorly in the liver (Fig. 5b).

(c) Patient $\mathrm{V}$ had a prominent right subcostal abscess pointing externally which was aspirated. 


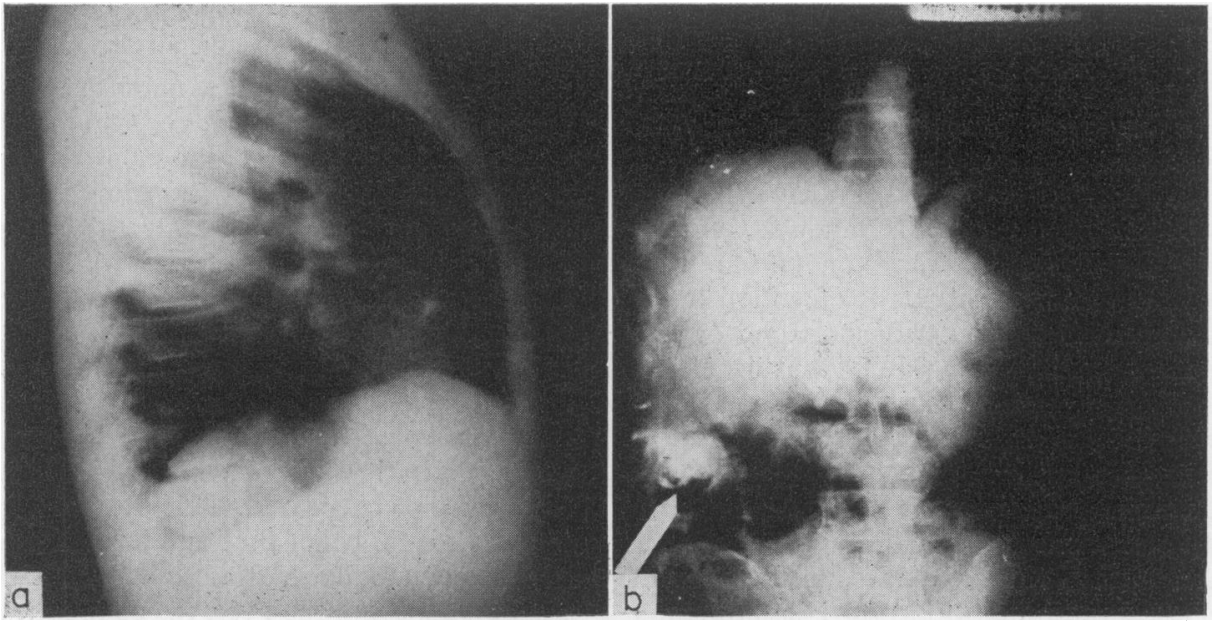

FIG. 5. (a) Patient M.A.I, showing an elevation of the diaphragm in the right lateral view. (b) Dye in the abscess cavity, confirming its position in the inferior aspect of the liver. Note elevation of diaphragm in the PA view (Group 1 pattern).
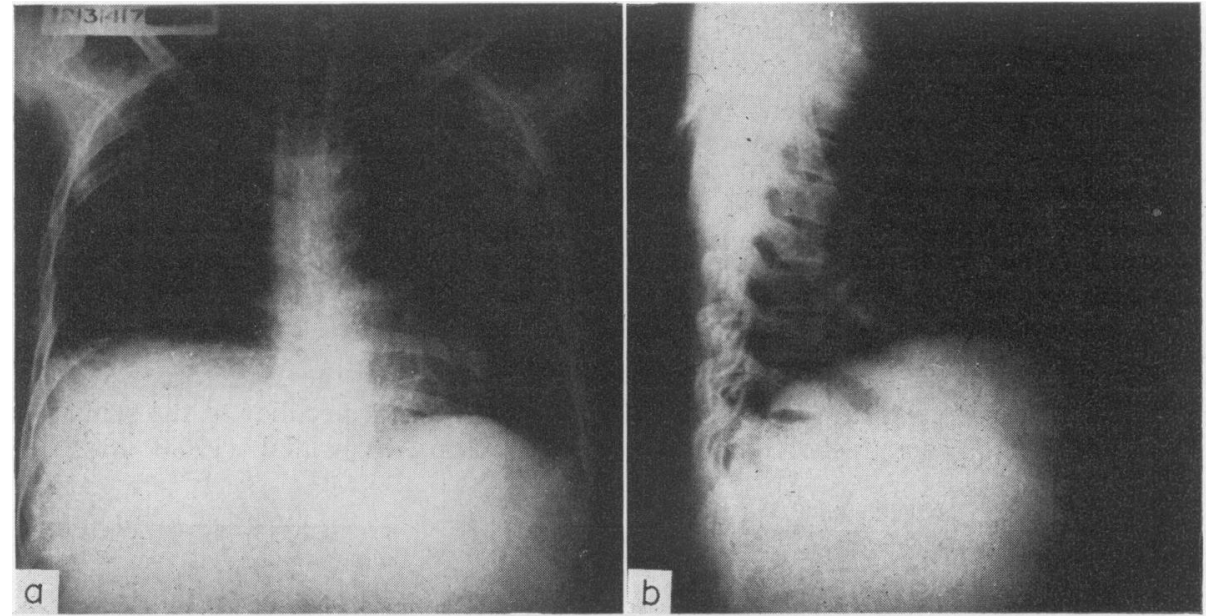

FIG. 6. Patient V. (a) Elevation of the diaphragm in PA and (b) right lateral view of a Group 1 pattern.

Radio-opaque dye was injected into the abscess cavity which revealed its situation in the liver. This patient showed a typical Group 1 radiological pattern with prominent elevation of the diaphragm in both views (Fig. 6a and b).

It thus appears that elevations of the right dome of the diaphragm could be associated with hepatic abscesses situated inferiorly in the liver or in the epigastric region.

\section{Discussion}

Elevation of the right dome of the diaphragm, a radiological feature of hepatic amoebiasis docu- mented in studies reported earlier was seen in a higher percentage of cases in this study. This in turn necessarily gave rise to a larger proportion of patients $(88 \%)$ who showed radiological abnormalities in their chest X-rays. This bigher incidence is probably due to one or other of two factors: (i) that the manifestations of the syndrome may be dependent on the geographical distribution or (ii) that lateral X-ray views were performed routinely in all the cases included in this study. No mention is made in the larger series reported earlier regarding the views of the chest taken. By doing lateral X-rays of the chest in all cases of hepatic amoebiasis, apart 
from increasing the incidence of the percentage of patients with abnormal X-ray findings, the presence of the interesting Group 2 patients was noted. The cause of this Group 2 radiological pattern with an absence of elevation of the right dome of the diaphragm in the PA views and significant elevation in the lateral views is obscure. DeBakey \& Ochsner (1951) and later Turrill \& Burnham (1966) have documented that the diaphragmatic elevation often involves the medial and anterior portions of the right hemi-diaphragm. It may well be that the elevation of the diaphragm in these Group 2 cases, by being placed more medially is obscured by a superimposed heart shadow.

It was a striking feature that even in the Group 1 patients, the elevations of the diaphragm were more prominently seen in the right lateral views. It is possible that the radiological Group 1 described, is preceded by the radiological Group 2 where the earliest abnormality of a significant elevation of the diaphragm in the right lateral view either localized or generalized is not accompanied by any elevation in the PA view.

Localized humping rather than a generalized elevation, a feature seen in this study, particularly in the Group 1 patients has also been documented by Schorr \& Schwartz (1951). It is not possible to say with any certainty whether this is due to an abscess situated in the superior aspect of the liver, as it was frequently seen in the patients with a good response to specific therapy and where pus was not demonstrated (Fig. 7a and b).

The observation that the cases with proved abscess also conformed to the radiologically distinct patterns seen in the cases where no pus was demonstrated was of interest. This is at variance with the views docu- mented earlier by Sepulveda et al. (1959) that there is a moderate elevation of the diaphragm in acute amoebic hepatitis while marked elevations or localized prominences are seen in acute amoebic abscesses. We observed that such clear-cut distinctions are incorrect, this observation being supported by case reports of hepatic abscesses with no significant radiological changes (Rowland, 1963). The case reports from this study also illustrate some points of interest: (1) that abscesses could be associated with any of the radiological patterns described and (2) that abscesses even placed inferiorly in the liver or epigastrically could produce significant elevations in the right dome of the diaphragm, either localized or generalized. This observation lends no support to the belief that an elevated diaphragm is often associated with an abscess placed in the right superior aspect of the liver, the commonest site for a liver abscess. The fact that the radiological patterns seen in this study are common to both groups, whether pus was demonstrated or not, and the observation that the Group 1 radiological type may be preceded by a Group 2 radiological pattern, supports our contention that all cases of hepatic amoebiasis probably belong to some part of a continuous spectrum of the syndrome culminating in the formation of large hepatic abscesses, single or multiple. This would in turn also indicate that ches radiology while being of importance in the diagnosis of the syndrome, is of only relative value in differenti ating clinically the cases where pus is demonstrable (hepatic abscesses) from the cases where no pus may be demonstrated.

Elevation in the right dome of the diaphragm however is not peculiar to the syndrome of hepatic amoebiasis. Rowland (1963) has described this

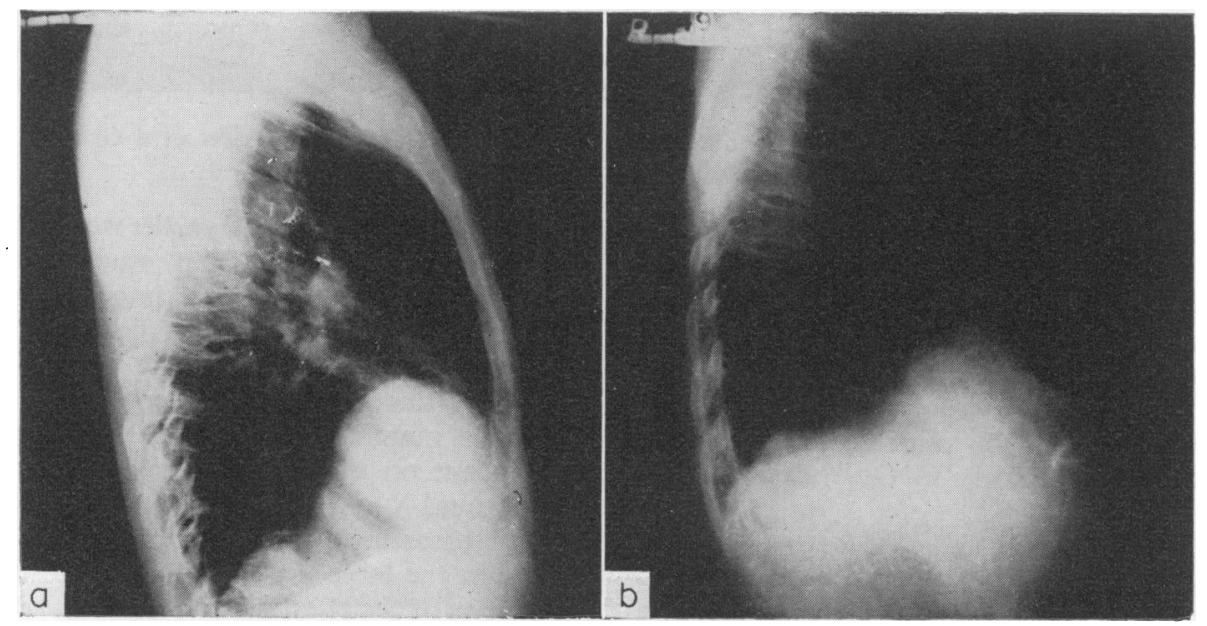

FIG. 7. Localized humping of the diaphragm. (a) No pus demonstrated, (b) pus demonstrated. 
abnormality in cases of primary carcinoma of the liver, while we have observed it in cases of hepatic cirrhosis usually with ascites, where the elevation of the diaphragm in the lateral views is often more striking. Thus radiological abnormalities should be assessed concurrently with the other criteria in the diagnosis of the syndrome of hepatic amoebiasis.

\section{References}

Amarjit Singh \& Jolly, S.S. (1962) Hepato-pulmonary amoebiasis in India. Journal of Tropical Medicine and Hygiene, 63, 71.

DeBakey. M.E. \& OChSNer, A (1951) Collective reviewhepatic amoebiasis, 20 year experience and analysis of 263 cases. International Abstracts of Surgery, 92, 209.

Islam, N., Allam, K.S. \& Quaderi, M.A. (1960) Hepatic amoebiasis. The Journal of Tropical Medicine and Hygiene, 63, 131.
KeAn, B.H. (1955) Amoebic hepatitis. Archives of Internal Medicine, 96, 667.

Lamont, N. McE. \& Pooler, N.R. (1958) Hepatic amoebiasis. Quarterly Journal of Medicine, 27, 390.

MuNK, J. (1944) X-ray appearance in amoebic hepatitis. British Journal of Radiology, 17, 48.

Rajasuriya, K. \& Nagaratnam, N. (1962) Hepatic amoebiasis in Ceylon. The Journal of Tropical Medicine and Hygiene, 65, 165.

RowLAND, H.A.K. (1963) Radiology of amoebic liver abscess. The Journal of Tropical Medicine and Hygiene, 66, 113.

Schorr, S. \& Schwartz, A. (1951) Roentgenologic manifestations of amoebiasis of the liver with concomitant findings in the chest. American Journal of Roentgenology, 66, 546.

SePulveda, B., Jinch, H., Bassols, F. \& Munoz, R. (1959) Amoebiasis of the liver. American Journal of Digestive Disorders, 4, 43.

TuRrill, F.L. \& Burnham, J.R. (1966) Hepatic amoebiasis. American Journal of Surgery, 111, 424. 